\title{
Nanostructuring polyetheretherketone for medical implants
}

\author{
Jasmin Althaus ${ }^{1-4}$, Celestino Padeste ${ }^{2}$, Joachim Köser ${ }^{3}$, \\ Uwe Pieles $^{3}$, Kirsten Peters ${ }^{4}$ and Bert Müller ${ }^{1, *}$ \\ ${ }^{1}$ Biomaterials Science Center (BMC), University of Basel, \\ c/o University Hospital Basel, 4031 Basel, Switzerland \\ ${ }^{2}$ Laboratory for Micro- and Nanotechnology, Paul Scherrer \\ Institute, 5232 Villigen, Switzerland \\ ${ }^{3}$ Institute of Chemistry and Bioanalytics, University of \\ Northwestern Switzerland, School of Life Sciences, \\ 4132 Muttenz, Switzerland \\ ${ }^{4}$ Department of Cell Biology, University of Rostock, \\ 18057 Rostock, Germany
}

\begin{abstract}
Surface roughness is a vital factor for medical implants since the cells of the surrounding tissue interact with the underlying substrate on the micro- and nanometer scales. In order to improve the surface morphology of implants, appropriate large-area micro- and nanostructuring techniques have to be identified being applicable to irregularly shaped structures. We demonstrate that plasma treatments of polyetheretherketone (PEEK) thin films produce nanostructured surfaces in a reproducible manner. They are easily tailored by varying plasma intensity using oxygen and ammonia as process gases. It was observed that roughness and nanostructure density linearly depend on plasma intensity. Oxygen plasma turned out to exhibit a stronger effect compared to ammonia plasma at the same processing conditions. For cell interaction studies, the mean size of the nanostructures was intentionally varied between $10 \mathrm{~nm}$ and $100 \mathrm{~nm}$. In vitro experiments revealed that human mesenchymal stem cells (hMSC) adhere inhomogenously on untreated PEEK films, but the plasma treatment with oxygen or ammonia allows the hMSC to adhere and proliferate. Fluorescence microscopy of the cells on the PEEK films turned out to be difficult because of the strong auto-fluorescence of the PEEK substrate. Stains including the whole cell vital stain Calcein-AM allowed cell morphology studies on plasma-treated PEEK films. In the case of the analysis of cell compartments such as the actin cytoskeleton, confocal laser scanning microscopy (CLSM) was successfully applied.
\end{abstract}

*Corresponding author: Dr. Bert Müller, Thomas Straumann Professor für Materialwissenschaft in der Medizin, Biomaterials Science Center (BMC), Universität Basel, c/o Universitätsspital, 4031 Basel, Schweiz

Phone: +4161 2659660

E-mail: bert.mueller@unibas.ch,www.bmc.unibas.ch

Previously published online January 19, 2012
Keywords: atomic force microscopy; auto-fluorescence; confocal laser scanning microscopy; human mesenchymal stem cells; nanostructures; oxygen and ammonia plasma treatments; polyetheretherketone (PEEK); Rat-2 fibroblasts.

\section{Introduction}

The surface chemistry, the surface roughness, and the stiffness of an implant play a crucial role in the biocompatibility of medical implants (1-3) because adherent cells interact with the accessible micro- and nanometer-size features (4-6). Titanium bone implants are usually sandblasted and etched to reach the necessary micro- and nanometer-scale roughness for appropriate osseointegration. For an increasing number of muscolo-skeletal applications polymer materials have been used, which exhibit the advantage of being radiolucent which is beneficial following the soft tissue formation around the implant. Their limited mechanical properties, however, restrict the materials selection. Therefore, the high-performance polymer polyetheretherketone (PEEK), which exhibits the desired mechanical properties, received the FDA-approval for trauma, orthopedic and spinal implants in 1982, and experiences an increasing use for medical implants and beyond. Besides reasonable mechanical properties it also exhibits outstanding chemical resistance, which makes it suitable as a biocompatible material and is already used in applications such as spinal disc cages and housings of pacemakers (7). For bone implants, PEEK surfaces have to be activated to allow for proper cell attachment. Plasma treatment belongs to the promising methods because of the ease of the process and the reproducible control of the final surface chemistry. We hypothesize that the plasma treatment can be exploited to generate and tailor nanostructures for improved osseointegration. Our hypothesis is also based on the recent observation of Dalby et al. (8), that patterns of nanostructures on polymeric substrates cause osteogenic differentiation of mesenchymal stem cells. They have found that, in contrast to highly ordered nanostructures, the randomly arranged nanostructures induce osteogenic differentiation. Therefore, we assume that the plasma etching, which leads to a random distribution of nanostructures on the PEEK surfaces, could be well suited for bone implants.

To study the cell-biomaterial interactions including cell morphology, fluorescence microscopy and confocal laser scanning microscopy (CLSM) are widely used. In the case of PEEK substrates, the application of fluorescence microscopy is critical because of the strong auto-fluorescence of the polymeric material. Hunter et al. (9), investigated the attachment and proliferation of osteoblasts and fibroblasts on biomaterials for orthopedic use, and explicitly excluded PEEK from the 
immuno-fluorescence study due to the prominent auto-fluorescence of the material. To overcome the problem, Briem et al. (10) replaced the immuno-fluorescence stains with Giemsa stains when investigating the response of primary fibroblasts and osteoblasts to plasma-treated PEEK. In order to address the problem of the auto-fluorescence, we have carefully analyzed the auto-fluorescence of commercially available PEEK films (APTIVTM Series from VICTREX, Hofheim, Germany) to explore the possible origin of the strong background fluorescence. One cause could arise from fluorescent additives. Therefore, PEEK has been chemically synthesized without any additive according to Risse et al. (11). Finally, we demonstrate that utilizing CLSM allows for the investigation of fluorescence-stained cells on micrometer-thin PEEK sheets with minimized background signal.

\section{Materials and methods}

\section{PEEK sheet pretreatment}

Hot embossing with a HEX03 press (JENOPTIK Mikrotechnik $\mathrm{GmbH}$, Germany) at a temperature of $160^{\circ} \mathrm{C}$ and a pressure of $100 \mathrm{kN}$ served to flatten commercially available amorphous APTIVTM PEEK films (Series 2000, Victrex Europa GmbH, Hofheim, Germany) with nominal thicknesses of $6 \mu \mathrm{m}, 12 \mu \mathrm{m}, 25 \mu \mathrm{m}$ and $50 \mu \mathrm{m}$ between two polished 4-inch silicon wafers.

\section{Plasma treatment}

Oxygen/argon or ammonia plasma treatments (Piccolo system, Plasma Electronic, Neuenburg, Germany) activated the embossed PEEK sheets. The embossing-marked films were placed at the bottom of the plasma chamber. Subsequently, the chamber was evacuated, flushed for a period of $5 \mathrm{~min}$ with a 2:1 mixture of oxygen/argon (200/100 sccm, 99.5/99.2\%, Messer, Lenzburg, Switzerland) or ammonia (200 sccm, 99.98\%, Messer, Lenzburg, Switzerland) and then equilibrated for further $5 \mathrm{~min}$ with oxygen/argon $(20 / 10 \mathrm{sscm})$ or ammonia $(30 \mathrm{sccm})$. The plasma treatments using a power of $10 \mathrm{~W}$, $25 \mathrm{~W}, 50 \mathrm{~W}, 75 \mathrm{~W}, 100 \mathrm{~W}, 125 \mathrm{~W}, 150 \mathrm{~W}, 175 \mathrm{~W}$, or $200 \mathrm{~W}$ always lasted $5 \mathrm{~min}$.

\section{Atomic force microscopy measurements (AFM)}

AFM measurements enabled us to determine the surface roughness and island density of plasma-activated PEEK sheets. Measurements were performed in TappingMode ${ }^{\circledR}$ in air under dry conditions on a Dimension IIIa instrument (Veeco, Mannheim, Germany) using silicon cantilevers with a $\mathrm{Si}_{3} \mathrm{~N}_{4}$ coating and a tip radius of $20 \mathrm{~nm}$, a spring constant of $40 \mathrm{~N} / \mathrm{m}$ and a resonance frequency of $325 \mathrm{kHz}$ (NSC15/A1BS, Mikromasch, CA, USA; manufacturers specifications). The scan area was set to $2 \times 2 \mu \mathrm{m}^{2}$. Data processing and roughness evaluation was done using the Nanoscope software. For each specimen the island density was determined from three individual characteristic square areas, each with approximately 100 islands.

\section{Transmission and fluorescence scans}

PEEK was synthesized according to Risse et al. (11). A tert-butyl substituted PEEK was prepared by nucleophilic substitution of tert- butylhydroquinone and 4,4'-difluorobenzophenone (Sigma-Aldrich). The tert-butyl substituent was cleaved via reversed Friedel-Crafts alkylation using trifluoromethansulfonic acid. The specially synthesized PEEK and corresponding starting materials in powder form were filled into 96-well plates until the bottom was covered.

The PEEK films were stamped out to fit into the 96-well plate format. The transmission measurements carried out in a 96-well quartz glass plate (Hellma, Müllheim, Germany) were recorded for wavelengths ranging from $240 \mathrm{~nm}$ to $1000 \mathrm{~nm}$. The fluorescence experiments in black 96-well plates were performed with a TECAN micro-plate reader infinite 200, equipped with a UV Xenon flash lamp (TECAN trading AG, Switzerland) using bottom reading with a detector gain of 60 . The excitation wavelengths were varied between $350 \mathrm{~nm}$ and $800 \mathrm{~nm}$ in steps of $10 \mathrm{~nm}$. The corresponding emission was acquired $30 \mathrm{~nm}$ above excitation wavelengths to 850 $\mathrm{nm}$ in $5 \mathrm{~nm}$ steps.

\section{Cell culture}

Rat-2 fibroblasts were cultured in DMEM medium under standard conditions $\left(5 \% \mathrm{CO}_{2}, 37^{\circ} \mathrm{C}\right)$. The fibroblasts were seeded over night on $70 \%$ ethanol sterilized class cover slips and PEEK sheets.

Human mesenchymal stem cells (hMSC) were isolated from liposuction-derived adipose tissue as described previously by Peters et al. (12) and seeded (96-well plate, $10^{4}$ cells $/ \mathrm{cm}^{2}$, triplicates) on the differently plasma-treated PEEK substrates for a period of 14 days. Cell cultivation was done under standard conditions.

\section{Fluorescence staining}

Cells were washed with phosphate buffered saline (PBS) and fixed with $4 \%$ paraformaldehyde in PBS for $10 \mathrm{~min}$ at room temperature. The cells were then permeabilized with $0.2 \%$ Triton X-100, washed, and incubated with a 1:40 dilution of mouse anti-human vinculin (Sigma-Aldrich, Buchs, Switzerland) and a 1:2000 dilution of TRITC-conjugated phalloidin (Sigma-Aldrich, Buchs, Switzerland) in PBS for $1 \mathrm{~h}$ at room temperature. Subsequently, the cells were labeled using a 1:1000 dilution of goat anti-mouse Alexa $488 \mathrm{sec}-$ ondary antibody (Invitrogen) in PBS during $30 \mathrm{~min}$. The cells were visualized on a BX-51 fluorescence microscope equipped with a fluorescence unit, and a Fluo-View 1000 confocal laser scanning microscope, both from Olympus (Hamburg, Germany).

hMSC were vital-stained with Hoechst 33324 (1:2000, SigmaAldrich, Taufkirchen, Germany) and Calcein-AM (Biomol GmbH, Hamburg, Germany; 1:200, ATT Bioquest) in DMEM medium during $15 \mathrm{~min}$ at a temperature of $37^{\circ} \mathrm{C}$. The staining medium was exchanged with fresh solution before imaging.

\section{Scanning electron microscopy (SEM)}

The plasma-treated PEEK films were coated with Au/Pd during $30 \mathrm{~s}$ using a current of $20 \mathrm{~mA}$ and applied a vacuum of $6 \mathrm{~Pa}$ (sputter coater Polaron, Thermo VG Scientific, Germany). Substrates were investigated with the field emission scanning electron microscope Supra 40 VP (Carl Zeiss, Jena, Germany) with an applied acceleration voltage of $10 \mathrm{kV}$ using the InLens detector.

\section{Results}

Plasma treatment is a common process to chemically activate polymer surfaces $(10,13-16)$, which is a prerequisite to 


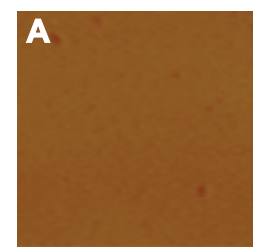

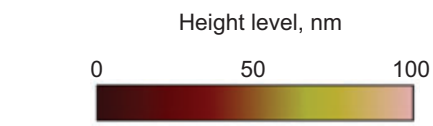
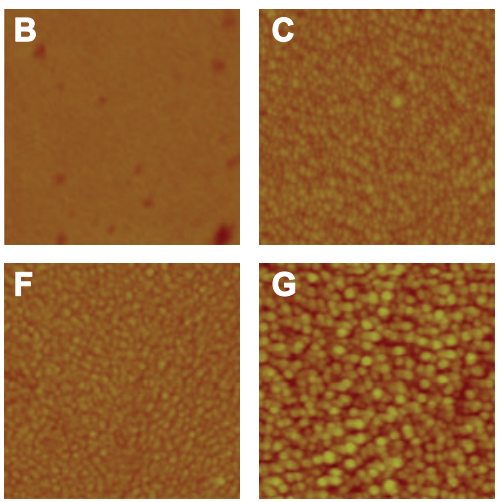
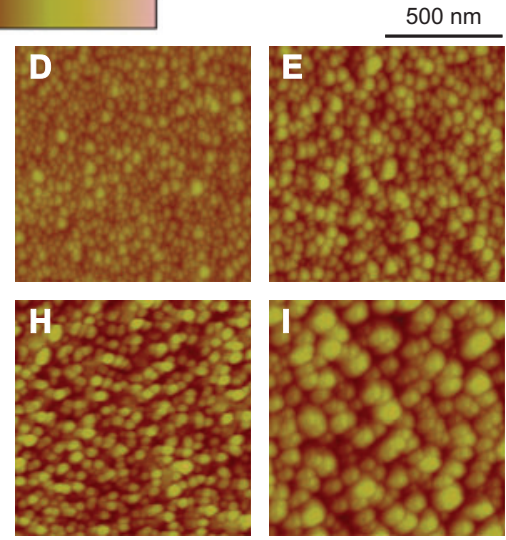

Figure 1 AFM images of plasma-treated PEEK films.

The $25 \mu \mathrm{m}$-thick films were plasma-treated for $5 \mathrm{~min}$. (A) untreated, (B-E) ammonia-plasma-treated, plasma power from left to right: $10 \mathrm{~W}, 50$ $\mathrm{W} 100 \mathrm{~W}$ and $200 \mathrm{~W}$. (F-I) oxygen-plasma-treated, plasma power from left to right: $10 \mathrm{~W}, 50 \mathrm{~W} 100 \mathrm{~W}$ and $200 \mathrm{~W}$.

achieve proper cell attachment. The process gases, oxygen and ammonia, modify the PEEK surface and lead to nanostructures as represented by the AFM images in Figure 1.

The plasma treatment with a duration of 5 min and the variation of the power between 10 and $200 \mathrm{~W}$ results in nanostructures of increasing size and PEEK surfaces with increasing roughness. This finding clearly indicates that plasma activation does not only change the chemical nature of the PEEK sheet but also induces significant changes in the surface morphology. These changes are also reflected in water contact angle measurements (data not shown). From the AFM measurements shown as an example in Figure 1, quantitative data were derived. The root-mean-square (RMS) roughness exhibits an almost perfectly linear behavior as a function of plasma power (see Figure 2A). The RMS roughness measurements of the oxygen plasma-treated surfaces show twice as large values to those of the ammonia treatments. Nevertheless, the island densities for the ammonia plasma treatments determined via area measurements of about 100 islands (island counting on certain areas), turned out to show values twice as high as the ones for the oxygen treatments (cp. Figure 2B).

The adipose tissue-derived hMSC, cultured on the differently treated PEEK substrates and the tissue culture polystyrene (TCPS) control for a period of 14 days, show a morphology characteristic for the plasma activation. While the hMSC on the PEEK sheet treated with a power of 10 $\mathrm{W}$ are similar to the ones on the control substrate, i.e., in a healthy state, the hMSC on the untreated PEEK have not properly adhered and spread out (see Figure 3). Also on harshly oxygen plasma-treated PEEK, the cells did not adhere and spread.

In order to study the cells on the PEEK films, fluorescence stains were applied with the aim to visualize the integrin- mediated focal adhesions. Integrins are heterodimeric cell membrane spanning receptors and connect the extracellular matrix (ECM) with the actin cytoskeleton of the cell.
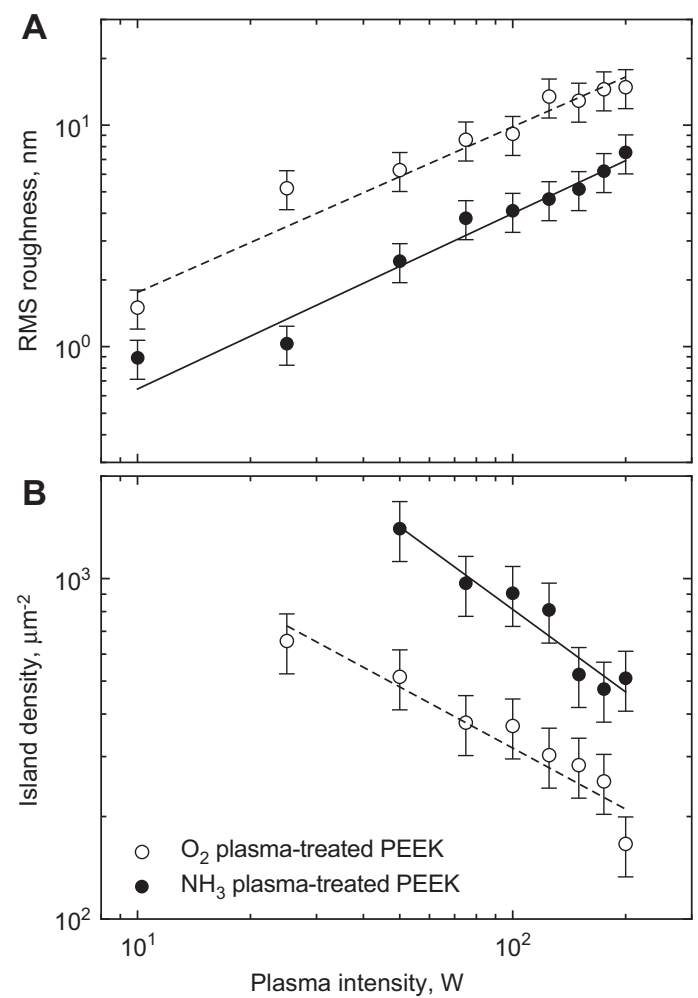

Figure 2 RMS roughness and island density of plasma-treated PEEK films.

The $25 \mu \mathrm{m}$-thick films were plasma-treated for $5 \mathrm{~min}$. 

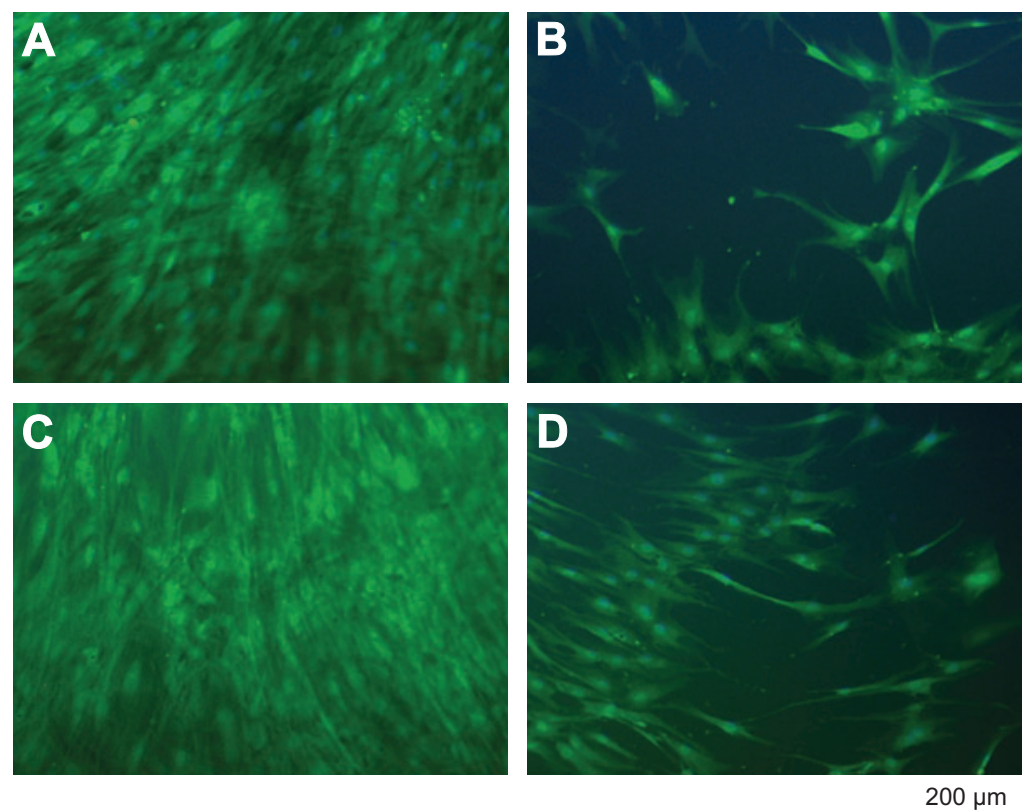

Figure 3 hMSC on oxygen-plasma-treated PEEK films.

hMSC from adipose tissue were cultured for 14 days under standard conditions. The $25 \mu \mathrm{m}$-thick films were plasma-treated for 5 min. (A) TCPS control, (B) untreated, (C) oxygen plasma power $10 \mathrm{~W}$, (D) oxygen plasma power $200 \mathrm{~W}$.

Here, the intracellular part of the integrin receptor binds to a protein complex including vinculin, which itself directly binds to actin. Therefore, co-localization of actin and vinculin is regarded as proper verification for the presence of focal adhesions (17). Rat-2 fibroblasts were seeded on APTIVTM PEEK sheets of different thicknesses and stained for vinculin and actin, giving rise to green- and red-colored features, respectively. Figure 4 shows the related images obtained with conventional epifluorescence microscopy and CLSM.

The images displayed in the first row of Figure 4 show the controls (rat-2 fibroblasts on glass slides). Here, the background is low and the green vinculin stain (FITC-labeled) clearly points to the focal adhesions of the cells. The redcolored actin cytoskeleton (TRITC-labeled) spans over the entire individual cells. The APTIVTM PEEK sheets exhibit a strong background fluorescence that seriously complicates the feature extraction using conventional fluorescence microscopy. This background fluorescence linearly increased with the film thickness. Hence, three-dimensional structures made out of PEEK, e.g., medical implants, can hardly be included into such fluorescence studies that strongly depend on the focal plane. In the model system studied, replacing the conventional fluorescence microscopy by CLSM, the background could significantly be reduced (see Figure 4). The characteristic pattern of the fluorescence stained cytoskeletal elements was obvious and similar to the control. The flexible $6 \mu \mathrm{m}$-thin PEEK films, however, exhibit an inhomogeneous background associated with their non-wavy surface. Therefore, one can reasonably assume that the investigation of cells on non-planar PEEK implant surfaces becomes complicated.
In order to characterize the optical properties of the PEEK films, the transmission spectra have been recorded (see Figure 5) for the four selected thicknesses.

With the exception of the Fabry-Pérot fringes that are very obvious for the $6 \mu \mathrm{m}$-thin sheets, the maximum transmission corresponds to about $80 \%$. Below $370 \mathrm{~nm}$, the PEEK sheets are opaque.

The background of the fluorescence images implies characteristic auto-fluorescence of PEEK. To obtain a detailed view about the auto-fluorescence of the PEEK sheets, spectroscopic fluorescence measurements for wavelengths ranging from 350 to $850 \mathrm{~nm}$ have been recorded and are presented in the diagrams of Figure 6.

To exclude that the fluorescence originates from any polymer additive, the PEEK polymer was synthesized according to the description of Risse et al. (11) and compared with the commercially available APTIVTM PEEK films from VICTREX. As shown in Figure 6, the PEEK films and the pure PEEK exhibit very similar emission spectra. Consequently, the auto-fluorescence is an inherent property of the PEEK polymer.

Strong emission resulted upon excitation at wavelengths between 370 and $550 \mathrm{~nm}$ with a broader maximum for excitation wavelengths between 370 and $450 \mathrm{~nm}$. Oxygen and ammonia plasma treatments did not change the emission profiles. The materials for the PEEK synthesis, $4,4^{\prime}$-difluorobenzophenone and tert-butylhydroquinone, were analyzed concerning fluorescence emission as well. While 4,4'-difluorobenzophenone showed fluorescence behavior similar to PEEK, tert-butylhydroquinone did not emit light in the excitation wavelength range between 400 and $800 \mathrm{~nm}$. 
Fluorescence microscopy
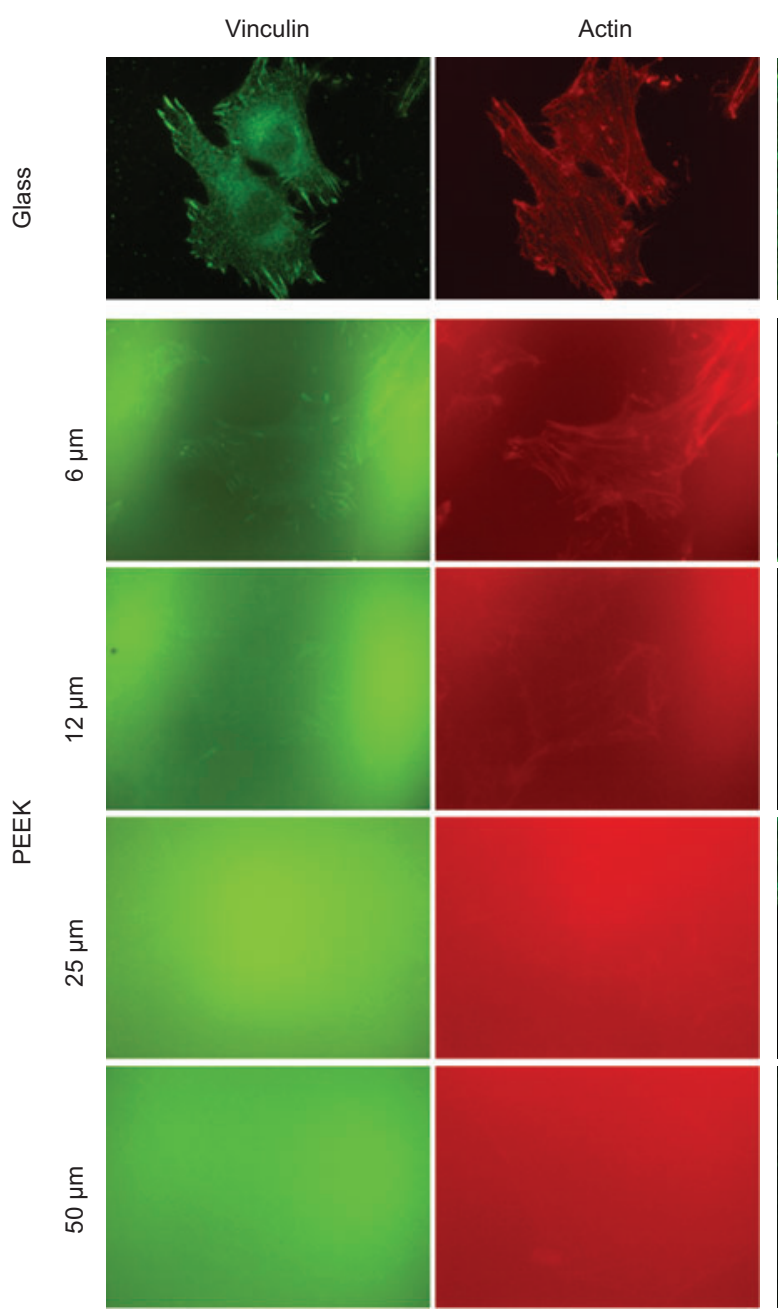

Actin

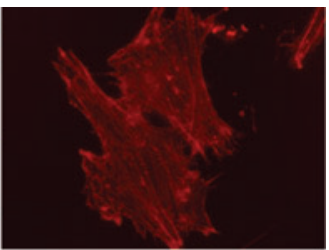

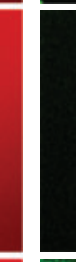
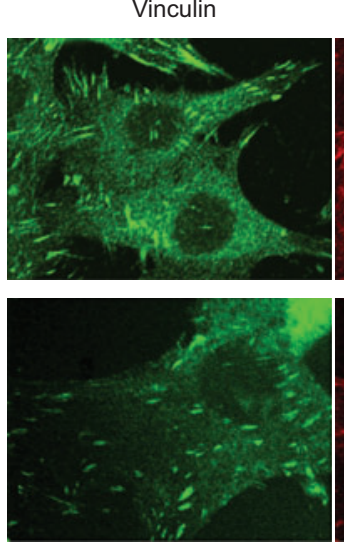

CLSM
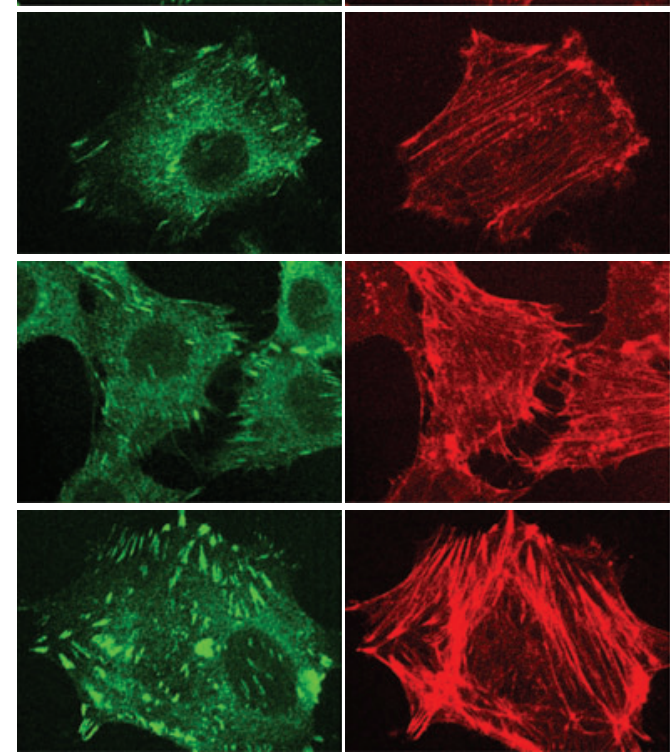

$20 \mu \mathrm{m}$

Figure 4 Rat-2 fibroblasts seeded on glass and differently thick APTIVTM PEEK films.

Actin stain: phalloidin-TRITC, vinculin stain: monoclonal anti-vinculin and goat-anti-mouse A-488.

\section{Discussion and conclusions}

The observed nanostructuring of PEEK by oxygen and ammonia plasma treatments is likely due to etching. To exclude a thermal effect, as the temperature within the plasma chamber increases during the processing by several Kelvin, we have performed an additional experiment comparing the nanostructures of a 20-min treatment with a four times 5 min processing. After each 5-min treatment, we have opened the chamber to harvest a part of the PEEK sheet for scanning electron microscopy imaging. Figure 7 displays these images, which do not show any significant difference between step-wise and the one-step processes.

Therefore, one can conclude that thermal effects are negligible. We speculate that the local etching rate depends on the molecule orientation within the semi-crystalline polymer. When etching is described as a negative growth, similar to the facet-depending growth rates in single-crystalline solids, the orientations with fast etching rates gradually disappear, which

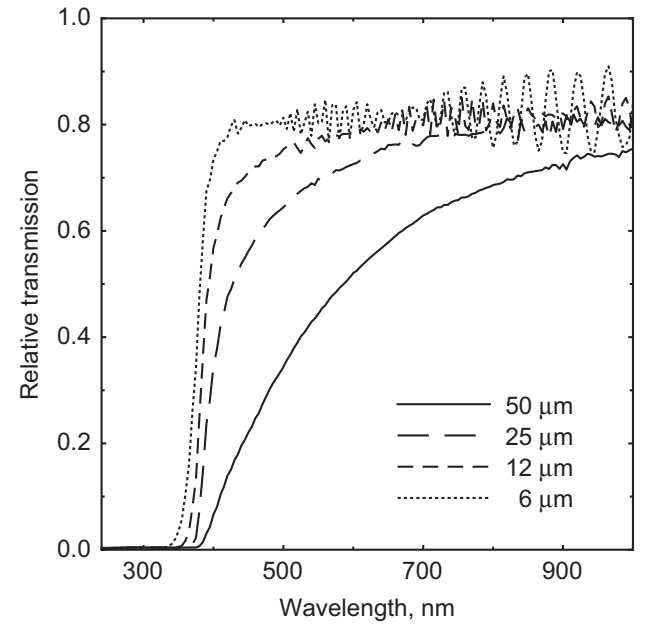

Figure 5 Transmission spectra of PEEK films. (Film thickness indicated.) 


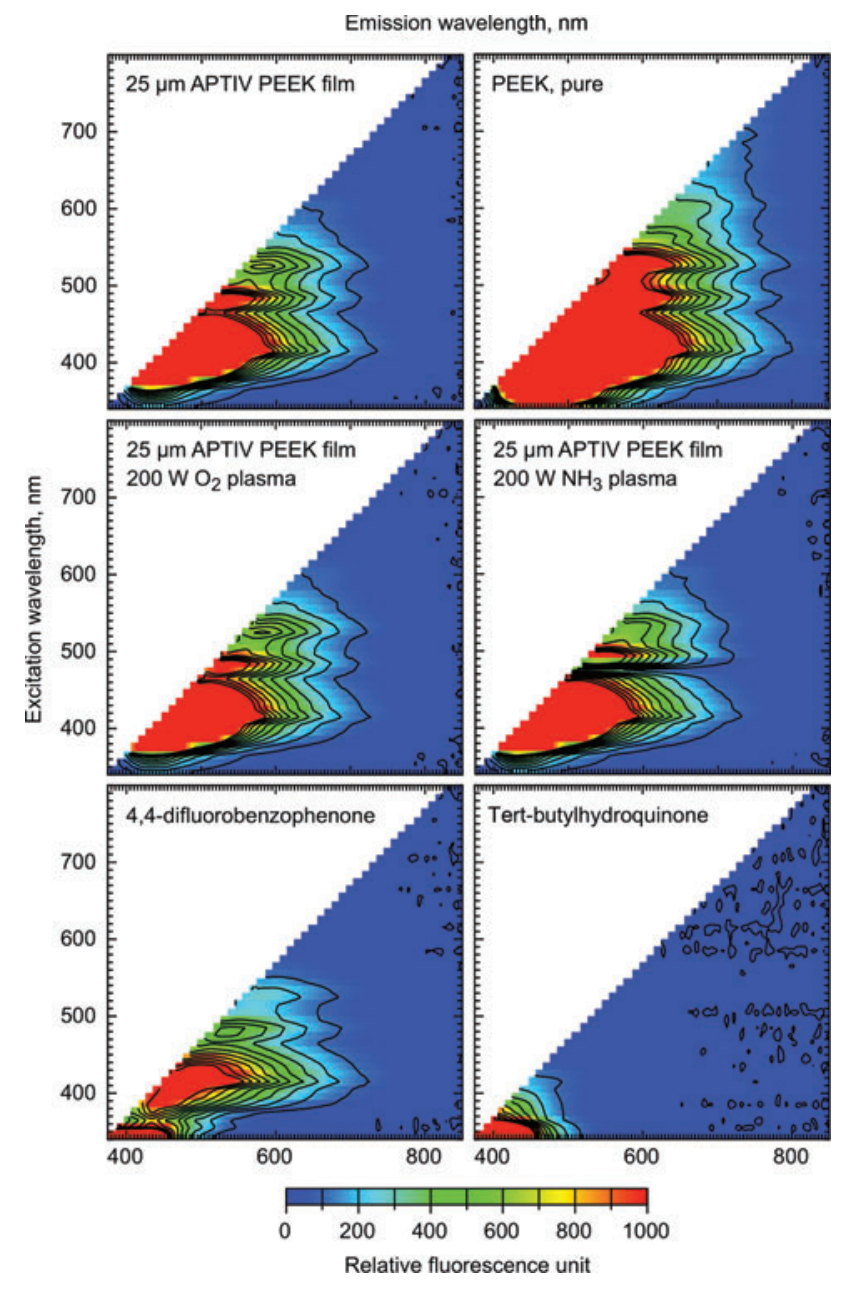

Figure 6 Fluorescence spectra from APTIVTM PEEK films, synthesized PEEK and starting materials.

Specimens were excited with wavelengths ranging from $350 \mathrm{~nm}$ to $800 \mathrm{~nm}$ in $10 \mathrm{~nm}$-steps. Emission spectra up to a wavelength of $850 \mathrm{~nm}$ were recorded $30 \mathrm{~nm}$ above excitation wavelength $(5 \mathrm{~nm}$ steps). The $25 \mu \mathrm{m}$-thick films were plasma-treated for $5 \mathrm{~min}$.

is linked to the formation of larger and larger nanostructures. Oxygen plasma exhibits a higher etching rate than ammonia plasma. This phenomenon can be explained, because the two plasma-activated reaction gases exhibit a different level of chemical reactivity causing the difference in the etching rate. Consequently, the nanostructures on the PEEK sheets can be tailored using the plasma power, the duration of processing, and the choice of the process gas.

AFM measurements directly provide data on the substrate roughness. In many experiments one observes, however, that the roughness of nanostructured surfaces depends on the scanning speed and the choice of the scanning range (18). Counting the nanometer-size features is rather independent on the spatial resolution of the imaging method and therefore a reliable parameter to compare specimens from different batches (19). Hence, we recommend selecting the process parameters via the nanostructure density rather than using RMS values derived from AFM data.
Surface nanostructures have a vital influence on protein adsorption and cellular response, making them a key parameter in characterizing the interactions between biological systems and man-made biomaterials (4, 19-21). In contrast to metallic and ceramic biomaterials, however, nanostructuring polymer materials is still poorly understood, although this group of biomaterials has numerous advantages and the plasma-induced roughness increase of polymers has been known for more than a decade (22). First, the plasma treatment can be realized on large areas, as required for the treatment of medical implants. Second, it can be combined with other structuring technologies such as soft lithography, replica molding or polymer de-mixing (23). Third, the plasma treatment generates random nanostructures, which seem to be favored by hMSC for osteogenic differentiation over highly ordered structures as recently described by Dalby et al. (8). Fourth, plasma treatment is a fast, dry, and reproducible process and easy to apply on large scales and therefore suitable for mass production. As a consequence, this method enables the production of large substrate areas necessary for in vitro cell culture studies with primary cells like hMSC to reach statistically significant statements. However, one drawback of the plasma-induced activation (chemical modification) is the creation of instable surfaces, which change their properties including the water contact angle over time, due to decay of the reactive molecular species remaining.

A further drawback of optimizing PEEK for medical implants is the optical imaging of cells on PEEK surfaces due to auto-fluorescence $(9,10)$. The detailed investigation of the fluorescence of commercially available PEEK films (VICTREX) and pure PEEK revealed that the broadband auto-fluorescence is an inherent material property and not caused by any additive. As the aromatic ring structures of the PEEK monomers consist of highly delocalized electrons, they are expected to be excitable to a greater extent than tightly bound electrons. According to the results of the fluorescence analysis, fluorescent dyes that emit in the UV and infrared spectral regions should be less prone to background fluorescence interference. Therefore, Alexa Fluor 350 and Alexa Fluor 633 are promising candidates for fluorescence microscopy on PEEK substrates, since the corresponding filters are less common but available for most fluorescent microscopes. Due to the relatively long wavelength of $633 \mathrm{~nm}$, a loss in resolution has to be taken into account. Notably, the cell live stain Calcein-AM, which has its emission maximum at 488 $\mathrm{nm}$, can be applied to visualize cells on PEEK films. The cell stain is strong compared to a cell compartment stain and a cell has a height of several micrometers, shifting the focal plane away from the auto-fluorescent PEEK surface.

PEEK belongs to the promising biomaterials for a variety of medical implants. To reach cell attachment and the desired cell response, the surface has to be activated. Plasma treatment is a suitable method to activate the PEEK surface, associated with the generation of nanostructures. Their density and size can be tailored by the choice of the process gas, the plasma power, and the duration of the plasma treatment. In summary our results guide a path towards improving the biocompatibility of PEEK implants. 

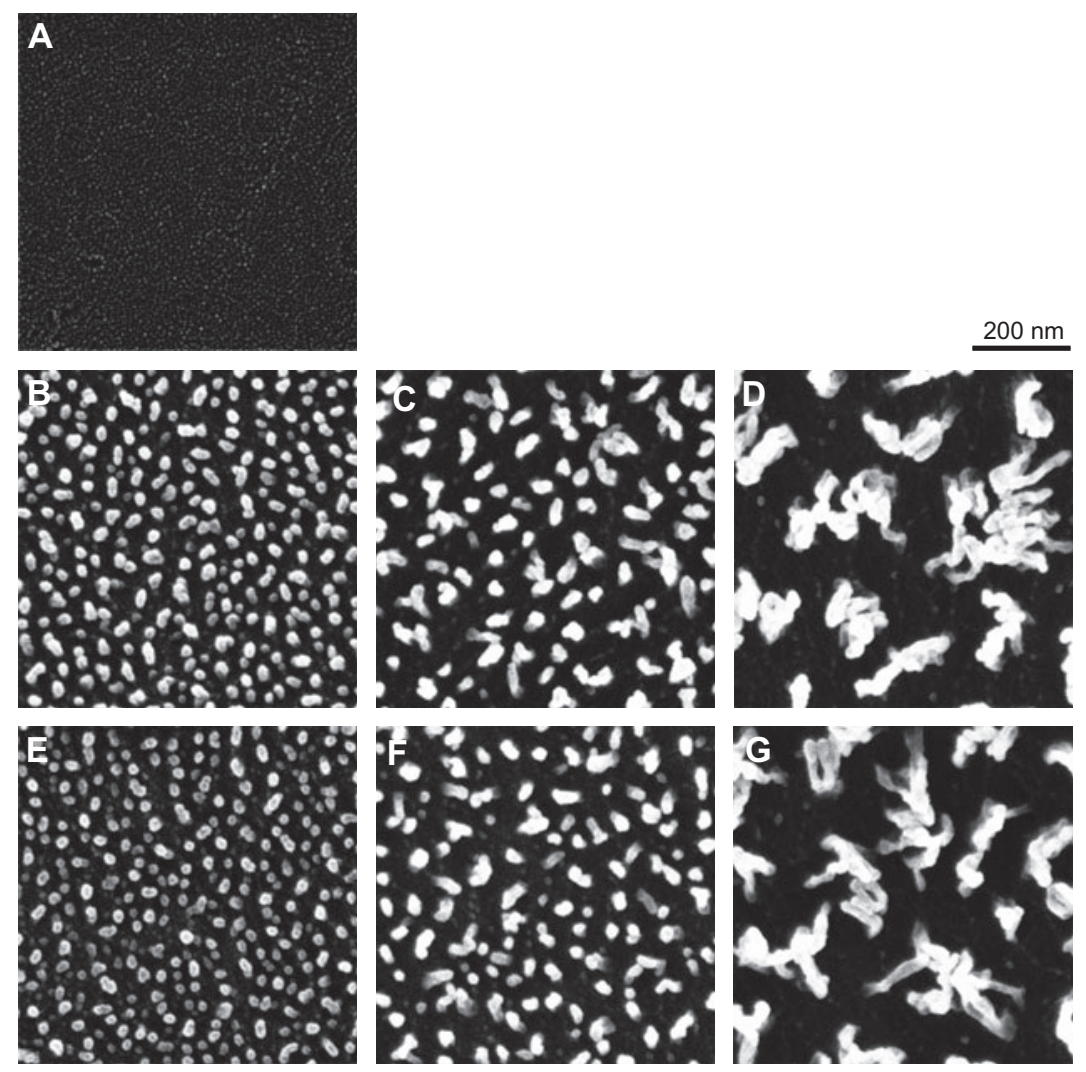

Figure 7 SEM images of $25 \mu \mathrm{m}$-thin PEEK films after $50 \mathrm{~W}$ oxygen plasma treatment with varying exposure time. (A) untreated, (B) $5 \mathrm{~min}$, (C) $10 \mathrm{~min}$, (D) $20 \mathrm{~min}$, (E) $5 \mathrm{~min}$, (F) $2 \times 5 \mathrm{~min}$ (G) $4 \times 5 \mathrm{~min}$.

\section{Acknowledgments}

We gratefully acknowledge Nadja Chiapparelli for the AFM measurements and Marcus Waser for the synthesis of PEEK. We thank Helmut Schift, Konrad Vogelsang and Mirco Altana for supporting us with knowledge about hot embossing and VICTREX for supplying us with APTIVTM PEEK films. Stefanie Adam kindly supported the cell culture work. Financial support was provided by grants from the Swiss Nanoscience Institute (project 6.2), the Rectors Conference of the Swiss Universities (CRUS), the European Union and the Federal State of Mecklenburg-Vorpommern, Germany (ESF/IV-WM-B340006/08).

\section{References}

1. Ponche A, Bigerelle M, Anselme K. Relative influence of surface topography and surface chemistry on cell response to bone implant materials. Part 1: physico-chemical effects. Proc Inst Mech Eng H 2010;224:1471-86.

2. Anselme K, Ponche A, Bigerelle M. Relative influence of surface topography and surface chemistry on cell response to bone implant materials. Part 2: biological aspects. Proc Inst Mech Eng H 2010;224:1487-507.

3. Engler AJ, Sen S, Sweeney HL, Discher DE. Matrix Elasticity Directs Stem Cell Lineage Specification. Cell 2006;126:677-89.

4. Riedel M, Müller B, Wintermantel E. Protein adsorption and monocyte activation on germanium nanopyramids. Biomaterials 2001;22:2307-16.
5. Variola F, Brunski JB, Orsini G, Tambasco de Oliveira P, Wazen R, Nanci A. Nanoscale surface modifications of medically relevant metals: state-of-the art and perspectives. Nanoscale 2011;3: 335-53.

6. Dohan Ehrenfest D, Coelho P, Kang B, Sul Y, Albrektsson T. Classification of osseointegrated implant surfaces: materials, chemistry and topography. Trends Biotechnol 2010;28:198-206.

7. Kurtz SM, Devine JN. PEEK biomaterials in trauma, orthopedic, and spinal implants. Biomaterials 2007;28:4845-69.

8. Dalby MJ, Gadegaard N, Tare R, Andar A, Riehle MO, Herzyk P, et al. The control of human mesenchymal cell differentiation using nanoscale symmetry and disorder. Nat Mater 2007;6:997-1003.

9. Hunter A, Archer CW, Walker PS, Blunn GW. Attachment and proliferation of osteoblasts and fibroblasts on biomaterials for orthopaedic use. Biomaterials 1995;16:287-95.

10. Briem D, Strametz S, Schröder K, Meenen NM, Lehmann W, Linhart W, et al. Response of primary fibroblasts and osteoblasts to plasma treated polyetheretherketone (PEEK) surfaces. J Mater Sci-Mater M 2005;16:671-7.

11. Risse W, Sogha DY. Synthesis of soluble high molecular weight poly(aryl1 ether ketones) containing bulky substitutents. Macromolecules 1990;23:4029-33.

12. Peters K, Salamon A, Van Vlierberghe S, Richly J, Kreutzer M, Neumann H-G, et al. A new approach for adipose tissue regeneration based on human mesenchymal stem cells in contact to hydrogels-an in vitro study. Adv Eng Mater 2007;11:B155-61.

13. Ha SW, Hauert R, Ernst KH, Wintermantel E. Surface analysis of chemically-etched and plasma-treated polyetherether- 
ketone (PEEK) for biomedical applications. Surf Coat Tech 1997;96:293-9.

14. Ha SW, Kirch M, Birchler F, Eckert KL, Mayer J, Wintermantel E, et al. Surface activation of polyetheretherketone (PEEK) and formation of calcium phosphate coatings by precipitation. J Mater Sci-Mater M 1997;8:683-90.

15. Cortese B, Morgan H. Controlling the wettability of hierarchically structured thermoplastics. Langmuir 2011; dx.doi.org/10.1021/ la203741b.

16. Tsougeni K, Vourdas N, Tserepi A, Gogolides E, Cardinaud C. Mechanisms of oxygen plasma nanotexturing of organic polymer surfaces: from stable super hydrophilic to super hydrophobic surfaces. Langmuir 2009;6:11748-59.

17. Geiger B. A $130 \mathrm{~K}$ protein from chicken gizzard: its localization at the termini of microfilament bundles in cultured chicken cells. Cell 1979;18:193-205.

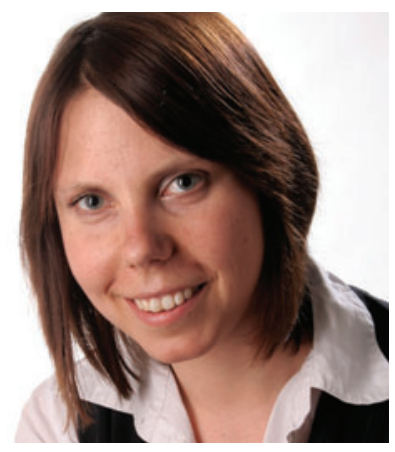

Jasmin Althaus received her diploma in chemistry from the University of Applied Sciences Northwestern Switzerland in 2004. After working at the Friedrich Miescher Institute in Basel as a research assistant in the group of Jan Hofsteenge, she moved on to the Biocenter in Basel where she earned her MSc in molecular biology in 2008. She is currently working towards her $\mathrm{PhD}$ degree in biomedical engineering on the effect of plasma-treated PEEK films on human mesenchymal stem cell differentiation at the University of Basel in close collaboration with Paul Scherrer Institute and University of Applied Sciences Northwestern Switzerland as well as the medical faculty of the University of Rostock.

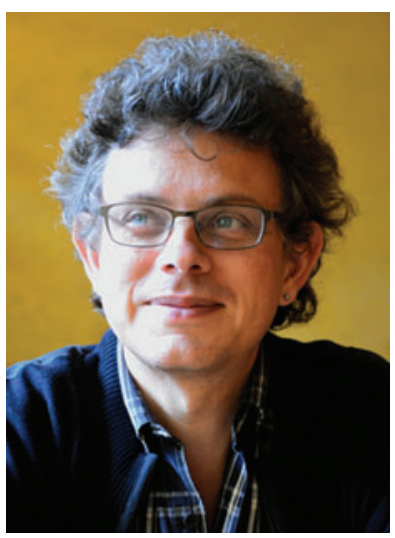

Celestino Padeste studied chemistry at the University of Zürich from where he received a $\mathrm{PhD}$ degree on inorganic solid state-gas phase reactions in 1989. After a Post-doc at the University of New South Wales in Sydney/Australia in the field of surface analysis of catalyst systems he was employed at the Micro- and Nanotechnology Laboratory at the Paul Scherrer Institute in Villigen/Switzerland. His work is focused on the design of functional surfaces by combination of nanostructuring methods including synchrotron radiation-based lithography with chemical surface modification, polymer grafting and protein immobilization.
18. Müller B, Riedel M, Michel R, Paul SMD, Hofer R, Heger D, et al. Impact of nanometer-scale roughness on contact-angle hysteresis and globulin adsorption. J Vac Sci Technol B 2001;19: 1715-20.

19. Müller B. Natural Formation of nanostructures: from fundamentals in metal heteroepitaxy to applications in optics and biomaterials science. Surf Rev Lett 2001;8:169-228.

20. Lord MS, Foss M, Besenbacher F. Influence of nanoscale surface topography on protein adsorption and cellular response. Nano Today 2010;5:66-78.

21. Latour R. Biomaterials: Protein-surface Interactions. Encyclopedia of Biomaterials and Bioengineering. 2008: pp. 27-284.

22. Chan CM, Ko TM, Hiraoka H. Polymer surface modification by plasmas and photons. Surf Sci Rep 1996;24:1-54.

23. Engel E, Michiardi A. Nanotechnology in regenerative medicine: the materials side. Trends Biotechnol 2008;26:39-47.

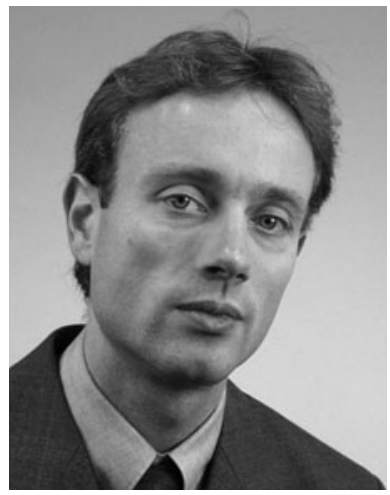

Joachim Köser received a diploma in biology from the University of Heidelberg. Following his $\mathrm{PhD}$ thesis at the German Cancer Research Center he moved to the Biocenter in Basel to work in the group of Ueli Aebi on the nuclear pore complex. Subsequently, he joined the spin-off company Concentris $\mathrm{GmbH}$ where he was responsible for the application development for cantilever sensors and now is working in the group of Uwe Pieles at the University for Applied Sciences Northwestern Switzerland in the field of medical nano-biotechnology.

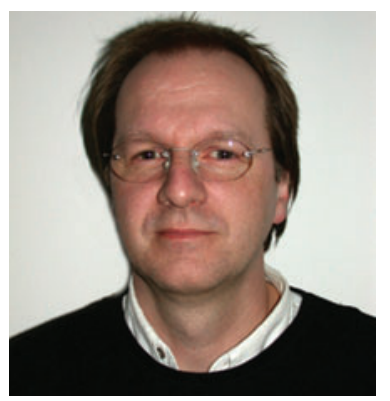

Uwe Pieles studied chemistry at the universities in Bielefeld and Göttingen (1981-1985) and obtained his diploma (1985) and the PhD degree (1988) at the Max-Planck Institute for experimental medicine under the supervision of Friedrich Cramer. Between 1989 and 1991 he worked as a postdoctoral researcher at the European Molecular Biology Laboratory. From 1991 to 1996 he worked as a laboratory head in the Central Research Laboratories of Ciba/Novartis in Switzerland (Basel). He became a group leader in the pharmaceutical research of Altana AG Germany (Konstanz, 1997-2000). Since 2000 he is a full professor and head of nanotechnology at the University of Applied Sciences Northwestern Switzerland in Muttenz. In 2007 he was rewarded with the Collano Prize. His main research activities include surface science, surface chemistry in materials science, chemical and biochemical sensors, and nanoparticles in catalysis and biochemical applications. 


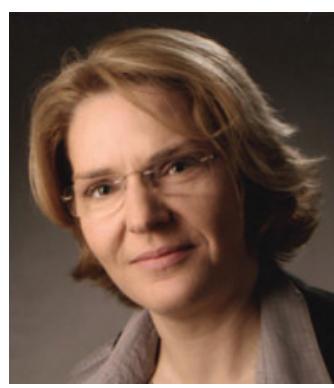

Kirsten Peters studied biology and did her diploma thesis (1993-1994) at the Westfälische WilhelmsUniversity in Münster, Germany, where she also received her $\mathrm{PhD}$ under the supervision of Jürgen Rauterberg (1994-1998). Between 1999 and 2006 she worked as a senior post-doctoral researcher at the Institute of Pathology at the Johannes Gutenberg-University Mainz (C. James Kirkpatrick). Since 2006 she is the head of the Junior Research Group (Department of Cell Biology, University of Rostock, Germany). In 2008 she received her habilitation in molecular medicine from the Johannes Gutenberg-University in Mainz.

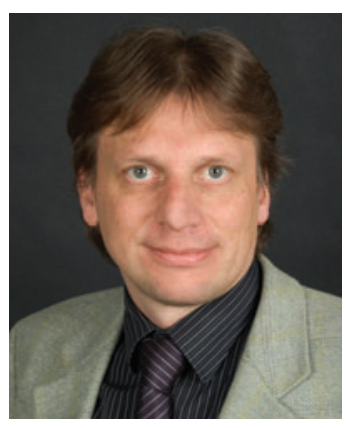

Bert Müller received a diploma in mechanical engineering (1982), followed by an MSc from the Dresden University of Technology and the $\mathrm{PhD}$ from the University of Hannover, Germany in 1989 and 1994, respectively. From 1994 to 2001, he worked as a researcher at the Paderborn University, Germany, EPF Lausanne, ETH Zurich. He became a faculty member of the Physics Department at ETH Zurich in April 2001. After his election as Thomas Straumann-Chair for Materials Science in Medicine at the University of Basel, Switzerland and his appointment at the Surgery Department of the University Hospital Basel in September 2006, he founded the Biomaterials Science Center. He also teaches physics and materials science at the ETH Zurich and the Universities of Basel and Bern. 